\title{
T-Odd Anomalous Interactions of the Top-Quark at the Large Hadron Collider
}

\author{
Apurba Tiwari $(\mathbb{D}$ and Sudhir Kumar Gupta \\ Department of Physics, Aligarh Muslim University, Aligarh, UP-202002, India \\ Correspondence should be addressed to Sudhir Kumar Gupta; sudhir.ph@amu.ac.in
}

Received 15 October 2020; Revised 16 February 2021; Accepted 9 March 2021; Published 15 April 2021

Academic Editor: Jouni Suhonen

Copyright @ 2021 Apurba Tiwari and Sudhir Kumar Gupta. This is an open access article distributed under the Creative Commons Attribution License, which permits unrestricted use, distribution, and reproduction in any medium, provided the original work is properly cited. The publication of this article was funded by SCOAP ${ }^{3}$.

We study the effects of T-odd interactions of top-quark via the pair production of the top-quark in the semileptonic detection modes at the Large Hadron Collider by means of the T-odd observables constructed through the momenta of the observed decay products of the top (and anti-top)-quark for a wide range of CP-violating scale $\Lambda$. Estimates on sensitivities of the coupling strength of such interactions for $13 \mathrm{TeV}$ LHC energy with $\int \mathscr{L} d t=36.1 \mathrm{fb}^{-1}, 140 \mathrm{fb}^{-1}$ and for HL-LHC with $14 \mathrm{TeV}$ energy with integrated luminosities of $0.3 \mathrm{ab}^{-1}, 1 \mathrm{ab}^{-1}, 2 \mathrm{ab}^{-1}$, and $3 \mathrm{ab}^{-1}$ are also presented for $\Lambda$ ranging between $M_{W}$ and $2 \mathrm{TeV}$.

\section{Introduction}

The phenomenon of charge and parity violation which was originally discovered in the neutral kaon-system via measuring the oscillation probability of $K^{0}$ into $\bar{K}^{0}[1]$ is now well understood. It besides being a new effect, had provided the ground for further exploration not only as an independent phenomenon but also its relation with a phenomenon such as Leptogenesis [2-5], Baryogenesis [6], nature of the Higgs boson $[7,8]$ and Dark matter of the Universe [9-11]. The Standard-Model (SM) which is originally CP-symmetric could still allow a tiny amount of $\mathrm{CP}$-violation via the inter-generational mixing of the fermions having identical quantum numbers through CKM-matrices [12]. However such effects are not sufficient to provide a satisfactory explanation to the observations such as the finite though a tiny amount of the electric-dipole-moment of the neutron [13, 14], origin to which may lie in the violation of CPsymmetry in the strong sector. These, therefore, require one to explore the possible sources of $\mathrm{CP}$-violation beyond the Standard Model.

Guided with the aforementioned phenomenon, in the present article we explore the possibility of a modelindependent extension of the SM in the form of T-odd anomalous interactions of the top-quark with gluons in the context of top-pair production at the LHC with pre-existing data at $13 \mathrm{TeV}$ center-of-mass (C.M.) energy and the forthcoming $14 \mathrm{TeV}$ run for projected Luminosities of about $0.3 \mathrm{ab}^{-1}, 1 \mathrm{a}$ $\mathrm{b}^{-1}, 2 \mathrm{ab}^{-1}$ and $3 \mathrm{ab}^{-1}$ respectively.

The T-violating interactions of the top-quark have already been studied in the literature for a fixed CPviolating scale in the Refs. [15-34]; for example, CPviolation at future $e^{+} e^{-}$collider in $t \bar{t}$ production is investigated in Ref. [15], Ref. [16] considered CP-violation due to complex top-Yukawa coupling in $e^{+} e^{-} \longrightarrow h t \bar{t}$ at the future $e^{+} e^{-}$collider, Charge-asymmetries in $b \bar{b}$ pair from topquark decay were first analysed in Ref. [17], Ref. [18] studied the CP-violation using T-odd correlations in lepton plus jets channel, Ref. [19] explored the possibilities of CP-violation in a rare process of top decay $t \longrightarrow b \bar{b} c$, Ref. [20] examines the possible CP-violating effects due to one-loop corrections to the top pair production process in the complex MSSM with minimal flavor violation (MFV) at hadron colliders and Ref. [21] investigates the CP-violation in the decay of a single top-quark produced in the t-channels. Similar studies have been performed for effective anomalous $\mathrm{CP}$-violating couplings for the process $\gamma \gamma \longrightarrow t \bar{t}$ in Refs. [35, 36], at FLC in Ref. [37] and in the context of muon colliders in Refs. [38]. The present article explores the effect of such anomalous interactions for a wide range of $\mathrm{CP}$-violating scale and 

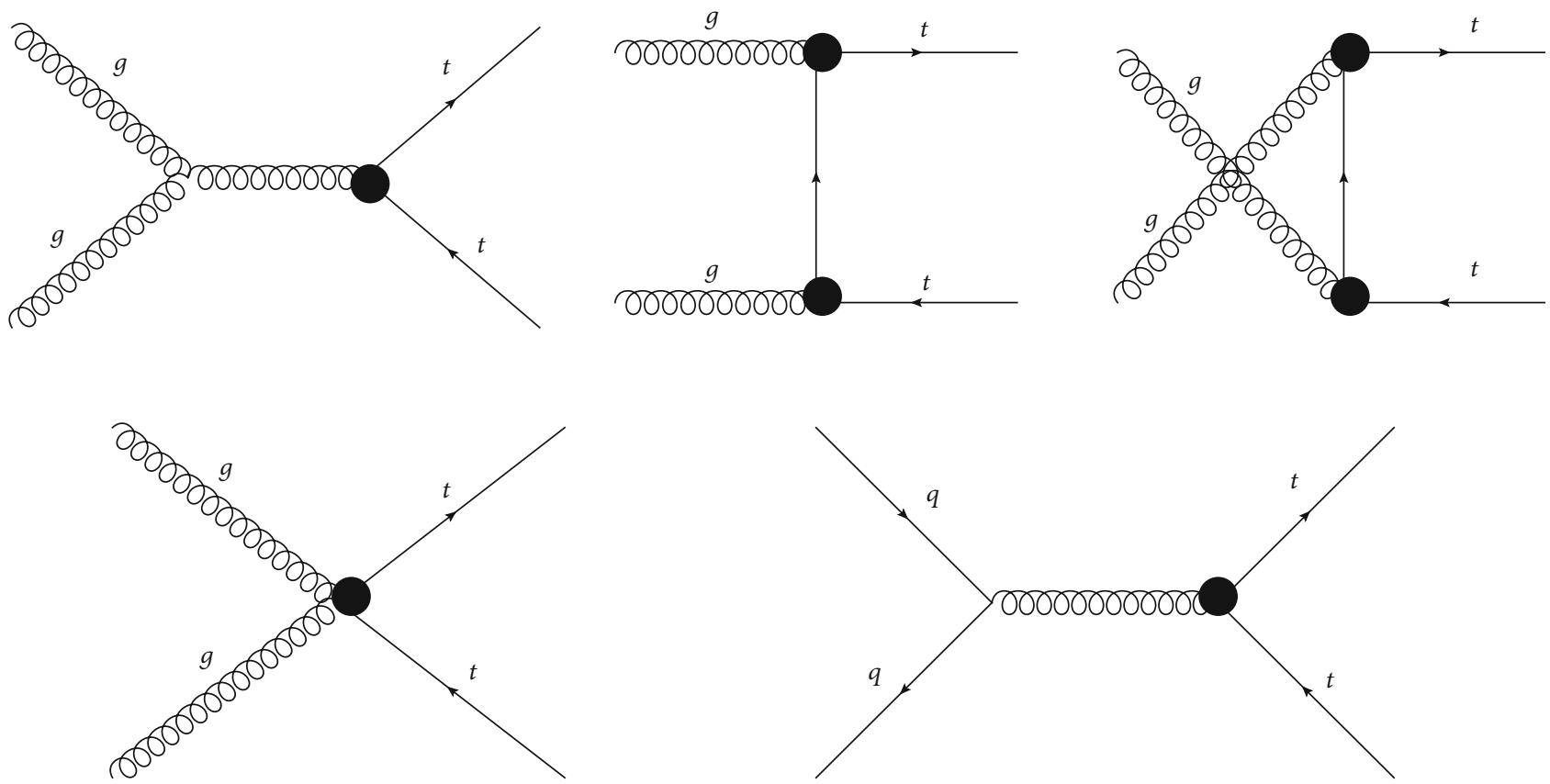

FIgURE 1: Feynman diagrams responsible for top-quark pair-production at the LHC.

provides the LHC-sensitivities for the coupling of such interactions via the process $p p \longrightarrow t \bar{t} \longrightarrow\left(b l^{+} v_{l}\right)\left(\bar{b} l^{-} \bar{v}_{l}\right)$ using Todd triple product correlations defined in Ref. [39].

Plan of the article is as follows: In Section 2 we discuss the model and possible T-odd observables for the top-pair production at the LHC and how these observables are suitable for analysing the effects of the $\mathrm{CP}$-violation. Section 3 discusses the numerical procedure and results on T-odd interactions. The experimental sensitivities of the T-odd couplings are also discussed in the same section. Finally, we summarise our findings in Section 4.

\section{T-Odd Observables and Top-Pair Production}

$\mathrm{CP}$-violation in the quark sector (except for the top-quark) faces an observational difficulty which partially lies in the fact that due to relatively larger life-time than the hadronisation scale, which is of about $140 \mathrm{MeV}\left(\simeq m_{\pi^{0}}\right.$, the mass of pion), quarks form bound states and thereby leave no scope for studying pure $\mathrm{CP}$-violation. By being much heavier than other quarks and also much energetic than the hadronisation scale, top-quark turns out to be the only expectation to test direct CP-violation in the quark sector. The life-time of a top-quark is less than the time required for a quark to hadronise therefore it does not form any bound state. Consequently the dynamics of top-production and decay do not get affected by complications of non-perturbative and bound state physics and, therefore, the $\mathrm{CP}$-violation effects involving top-quark will be of direct type. At hadron colliders, processes involving top-quarks have a further advantage in having larger cross-sections due to the strong interactions. This, therefore, enables us to directly investigate the effects of such interactions via the pair-production of the top-
TABLE 1: Experimental values of Standard Model input parameters [49].

\begin{tabular}{lc}
\hline SM parameter & Experimental value \\
\hline$m_{b}\left(m_{b}\right)$ & $4.7 \pm 0.06 \mathrm{GeV}$ \\
$m_{t}\left(m_{t}\right)$ & $173.0 \pm 0.4 \mathrm{GeV}$ \\
$M_{W}$ & $80.387 \pm 0.02 \mathrm{GeV}$ \\
$\alpha_{s}^{\bar{M} S}\left(M_{z}\right)$ & $0.118 \pm 0.001$ \\
\hline
\end{tabular}

quarks and their subsequent decays into a pair of leptons and $b$-quarks.

Our study of finding CP-violation is based on estimating asymmetries through $\mathrm{CP}$-violating observables. CP-odd observables can be formed using T-odd correlations which may not necessarily be CP-odd instead these could be CPeven as well and T-odd is not for time-reversal here, rather, it represents naive T-odd [40].

The chromo-electric dipole moment (CEDM) of the top-quark causes the $\mathrm{CP}$-violation in the top-pair production vertex. In the presence of T-odd interactions of the top-quark with gluon, the SM Lagrangian could be modified for the $t \bar{t}$ production process by the following interaction term [41]:

$$
\mathscr{L}_{\mathrm{int}}=-i \frac{g_{s}}{2}\left(\frac{d_{g}}{\Lambda}\right) \bar{t} \sigma_{\mu \nu} \gamma_{5} G^{\mu \nu} t
$$

with $g_{s}$ being the strong coupling constant, $G^{\mu v}$ the gluon fieldstrength tensor, $d_{g}$ and $\Lambda$ being the interaction strength and energy scale of the CP-violation respectively and $\sigma_{\mu \nu}=2 i\left[\gamma_{\mu}\right.$, 


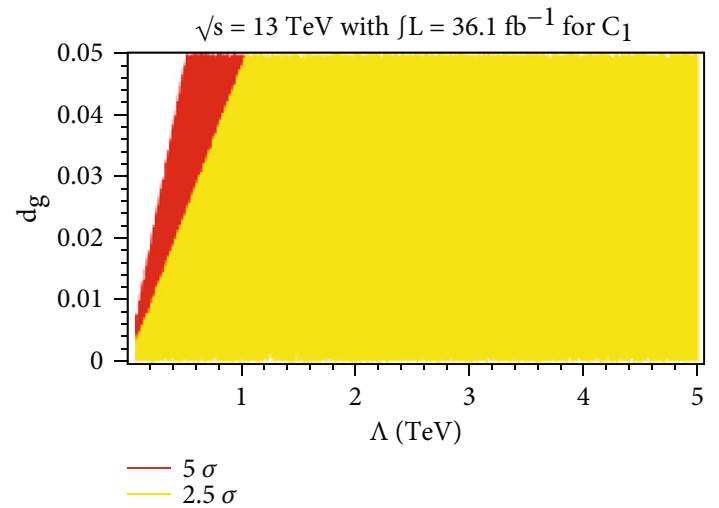

(a)

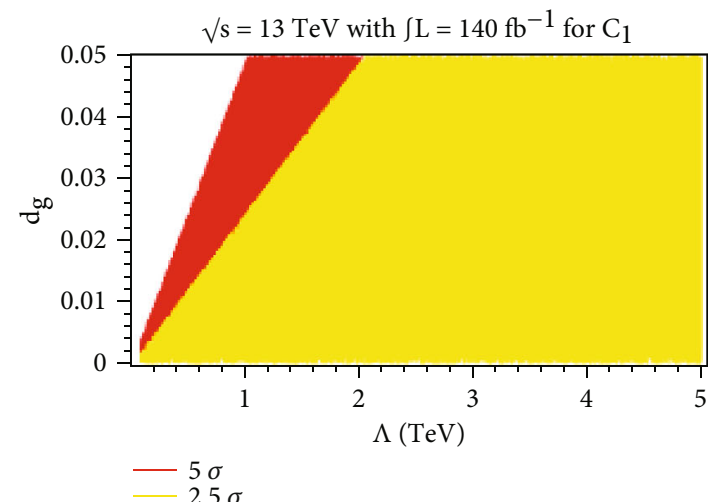

(b)

Figure $2: d_{g}$ vs. $\Lambda$ for observable $\mathscr{C}_{1}$ at $\sqrt{S}=13 \mathrm{TeV}$ energy at LHC for an integrated luminosity of (a) $36.1 \mathrm{fb}^{-1}$ and (b) $140 \mathrm{fb}^{-1}$ respectively.

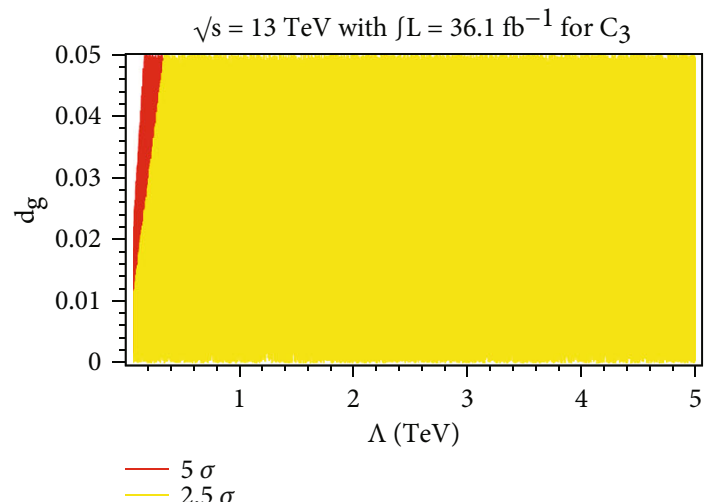

(a)

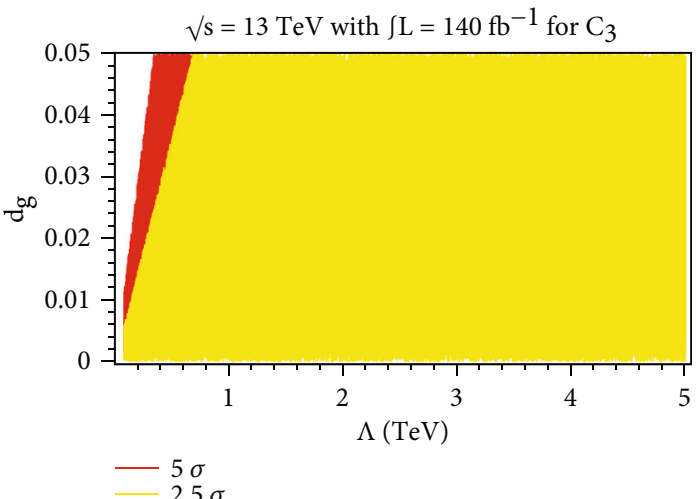

(b)

Figure 3: $d_{g}$ vs. $\Lambda$ for observable $\mathscr{C}_{3}$ at $\sqrt{S}=13 \mathrm{TeV}$ energy at LHC for an integrated luminosity of (a) $36.1 \mathrm{fb}^{-1}$ and (b) $140 \mathrm{fb}^{-1}$ respectively.

$\gamma_{\nu}$. The Lagrangian in Eq. (1) will give a new dimension five vertex $\bar{t} g g$ (which is absent in the SM) in addition to modifying the pre-existing $t \bar{t} g$ vertex. This new vertex $t \bar{t} g g$ is obviously CP-odd in nature according to the above equation.

These would clearly have a significant contribution to the top-pair production processes at hadron colliders, particularly for colliders alike LHC where the fusion of gluons emerging from the colliding protons makes about $90 \%$ contribution, the rest being the annihilation of light-partons of opposite charges. A schematic representation of various parton-level processes describing the production of $t \bar{t}$ at the LHC where the modification occurs due to the presence of additional T-odd interactions given by Eq. (1) is shown in Figure 1. The first four diagrams of Figure 1 represent the production of $t \bar{t}$ pairs through $g g$ fusion and the last one is via $q \bar{q}$ annihilation. The first three diagrams of Figure 1 are present in the SM as well, the fourth diagram which is absent in the SM represents the effective $t \bar{t} g g$ vertex and is the expandable SM. It is also worthwhile to mention that as the semileptonic decay of the top (anti-top) takes place due to weak-interactions, the branching ratio of the top-quark will remain intact as of the $S M$.

At first, we start our calculation with the T-odd correlations induced by anomalous top-quark couplings defined in the following equations:

$$
\begin{aligned}
& \mathscr{C}_{1}=\epsilon\left(p_{b}, p_{\bar{b}}, p_{l^{+}}, p_{l^{-}}\right) \\
& \mathscr{C}_{2}=\tilde{q} \cdot\left(p_{l^{+}}-p_{l^{-}}\right) \epsilon\left(p_{l^{+}}, p_{l^{-}}, p_{b}+p_{\bar{b}}, \tilde{q}\right) \\
& \mathscr{C}_{3}=\tilde{q} \cdot\left(p_{l^{+}}-p_{l^{-}}\right) \epsilon\left(p_{b}, p_{\bar{b}}, p_{l^{+}}+p_{l^{-}}, \tilde{q}\right) \\
& \mathscr{C}_{4}=\epsilon\left(P, p_{b}-p_{\bar{b}}, p_{l^{+}}, p_{l^{-}}\right) \\
& \mathscr{C}_{5}=\epsilon\left(p_{b}+p_{l^{+}}, p_{\bar{b}}+p_{l^{-}}, p_{b}+p_{\bar{b}}, p_{l^{+}}-p_{l^{-}}\right),
\end{aligned}
$$

where in the above expressions $\epsilon(a, b, c, d)=\epsilon_{\mu \nu \alpha \beta} a^{\mu} b^{v} c^{\alpha} d^{\beta}$ with $\epsilon_{\mu \nu \alpha \beta}$ being the Levi-Civita symbol of rank 4 which is completely anti-symmetric with $\varepsilon_{0123}=1$ and $p_{b}\left(p_{\bar{b}}\right), p_{l^{+}}\left(p_{l^{-}}\right)$ represent the four-momenta of $b(\bar{b})$-quark, lepton (anti-lepton) respectively. $P$ is the sum of four-momenta of $b$-quark, lepton, anti-b-quark and anti-lepton and $\tilde{q}$ is the difference of two-beam four momenta, defined as

$$
\begin{aligned}
& P=p_{b}+p_{l^{+}}+p_{\bar{b}}+p_{l^{-}} \\
& \tilde{q}=P_{1}-P_{2} .
\end{aligned}
$$

It is interesting to note that the aforementioned 


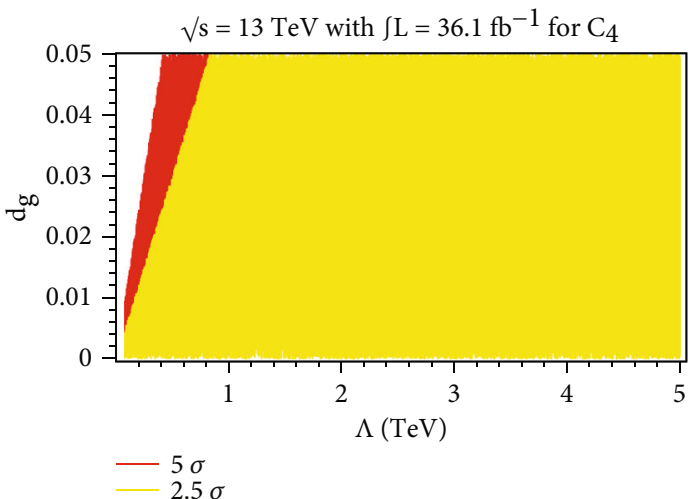

(a)

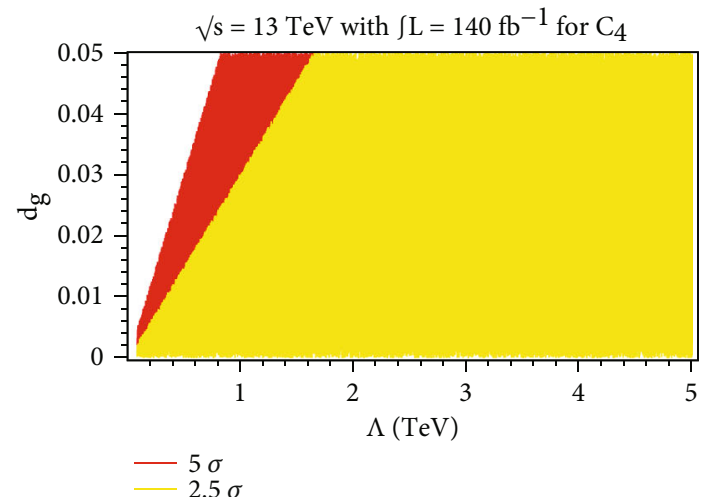

(b)

Figure 4: $d_{g}$ vs. $\Lambda$ for observable $\mathscr{C}_{4}$ at $\sqrt{S}=13 \mathrm{TeV}$ energy at LHC for an integrated luminosity of (a) $36.1 \mathrm{fb}^{-1}$ and (b) $140 \mathrm{fb}^{-1}$ respectively.

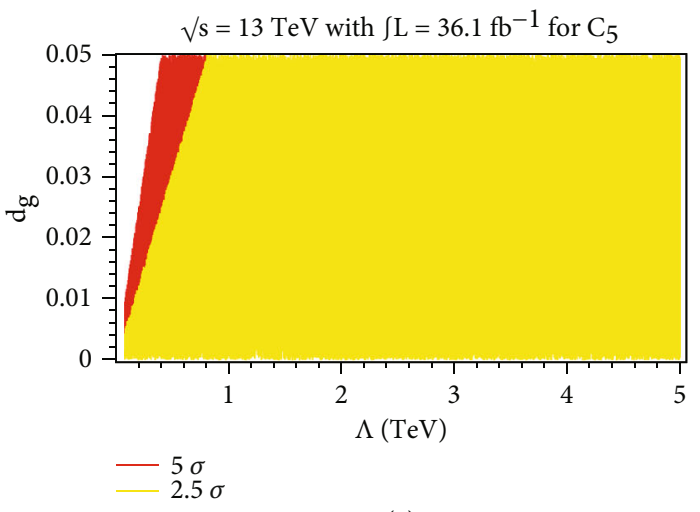

(a)

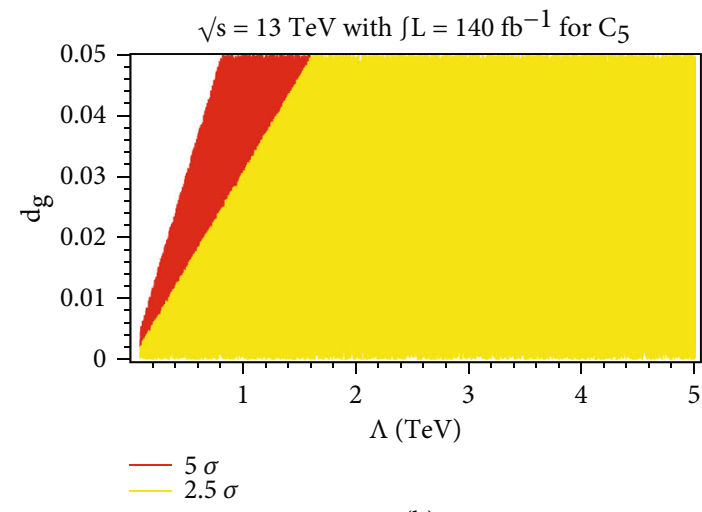

(b)

Figure 5: $d_{g}$ vs. $\Lambda$ for observable $\mathscr{C}_{5}$ at $\sqrt{S}=13 \mathrm{TeV}$ energy at LHC for an integrated luminosity of (a) $36.1 \mathrm{fb}^{-1}$ and (b) $140 \mathrm{fb}^{-1}$ respectively.

observables neither require reconstruction of the produced tops nor any information about the spin of the produced particles. Also, a $b$-jet is distinguished with a $\bar{b}$-jet by measuring the direction of leptons i.e. the $b$-jet closer to $l^{+}$is identified as the one arising due to a $b$-quark whereas the other $b$-jet closer to $l^{-}$is identified as the one arising due to a $\bar{b}$-quark.

Let us now consider observable $\mathscr{C}_{1}$ to check its CP properties [28, 39]

$$
\begin{aligned}
& \mathscr{C}_{1}=\epsilon\left(P_{b}, P_{\vec{b}}, P_{l^{+}}, P_{l^{\prime}}\right) \stackrel{(b \vec{b})_{\mathrm{CM}}}{\longrightarrow} \propto \vec{P}_{b} \cdot\left(\vec{P}_{l^{+}} \times \vec{P}_{l^{\prime}}\right) \\
& \text { Now } \vec{P}_{b} \cdot\left(\vec{P}_{l^{+}} \times \vec{P}_{l}\right) \stackrel{\mathrm{C}}{\rightarrow} \vec{P}_{\bar{b}} \cdot\left(\vec{P}_{l} \times \vec{P}_{l^{+}}\right)=-\vec{P}_{\bar{b}} \cdot\left(\vec{P}_{l^{+}} \times \vec{P}_{l^{\prime}}\right)=\vec{P}_{b} \cdot\left(\vec{P}_{l^{+}} \times \vec{P}_{l^{\prime}}\right) \\
& \vec{P}_{b} \cdot\left(\vec{P}_{l^{+}} \times \vec{P}_{l^{\prime}}\right) \stackrel{\mathrm{P}}{\rightarrow}-\vec{P}_{b} \cdot\left(-\vec{P}_{l^{+}} \times-\vec{P}_{l^{-}}\right)=-\vec{P}_{b} \cdot\left(\vec{P}_{l^{+}} \times \vec{P}_{l^{-}}\right) .
\end{aligned}
$$

In the above equation the left-hand side of the arrow describes the frame independent correlation and the righthand side represents the correlation in a particular C.M. reference frame. In the first line of Eq. (4), we go through $b \bar{b}$ C.M. frame which results in the triple product form. The obtained triple-product undergoes the charge conjugation and parity operation in the second and third lines, respectively, to ensure that it is CP-odd. Similarly, if we consider the $\left(l^{+} l^{-}\right)$C.M. frame, the above observable takes the following form $[28,39]$ :

$$
\begin{aligned}
& \mathscr{C}_{1}=\epsilon\left(P_{b}, P_{\bar{b}}, P_{l^{+}}, P_{\digamma}\right) \stackrel{\left(l^{+} \tau\right)_{\mathrm{CM}}}{\longrightarrow} \propto \vec{P}_{l^{+}} \cdot\left(\vec{P}_{b} \times \vec{P}_{\bar{b}}\right) \\
& \text { Now } \vec{P}_{l^{+}} \cdot\left(\vec{P}_{b} \times \vec{P}_{\bar{b}}\right) \stackrel{\mathrm{C}}{\rightarrow} \vec{P}_{l^{\prime}} \cdot\left(\vec{P}_{\bar{b}} \times \vec{P}_{b}\right)=-\vec{P}_{l^{\prime}} \cdot\left(\vec{P}_{b} \times \vec{P}_{\bar{b}}\right)=\vec{P}_{l^{+}} \cdot\left(\vec{P}_{b} \times \vec{P}_{\bar{b}}\right) \\
& \vec{P}_{l^{+}} \cdot\left(\vec{P}_{b} \times \vec{P}_{\bar{b}}\right) \stackrel{\mathrm{P}}{\rightarrow}-\vec{P}_{l^{+}} \cdot\left(-\vec{P}_{b} \times-\vec{P}_{\bar{b}}\right)=-\vec{P}_{l^{+}} \cdot\left(\vec{P}_{b} \times \vec{P}_{\bar{b}}\right) .
\end{aligned}
$$

This further suggests that $\mathscr{C}_{1}$ is indeed CP-odd. In addition to the observables discussed in Eqs. (2), we also construct the following new observables:

$$
\begin{aligned}
& \mathscr{C}_{6}=\epsilon\left(P, \tilde{q}, p_{l^{+}}+p_{b}, p_{l^{-}}+p_{\bar{b}}\right) \\
& \mathscr{C}_{7}=\epsilon\left(P, \tilde{q}, p_{l^{+}}, p_{l^{-}}\right) \\
& \mathscr{C}_{8}=\epsilon\left(P, \tilde{q}, p_{b}, p_{\bar{b}}\right) .
\end{aligned}
$$

The advantage of considering these additional observables lies in the fact that these require lesser information than the observables defined in Eqs. (2). For example, observable 


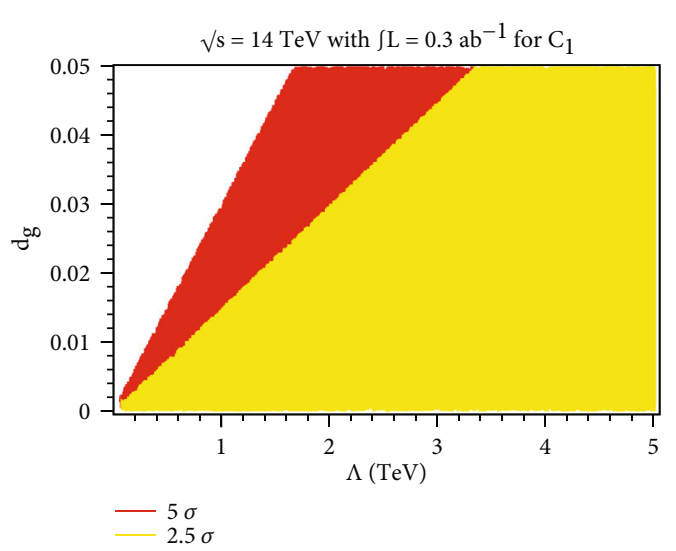

(a)

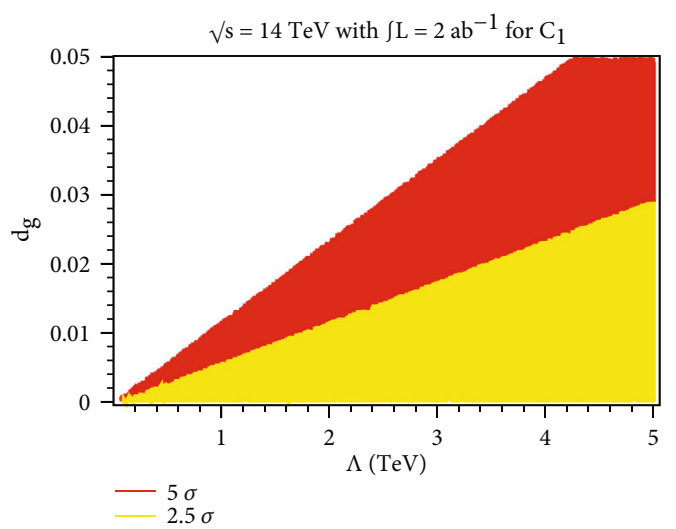

(c)

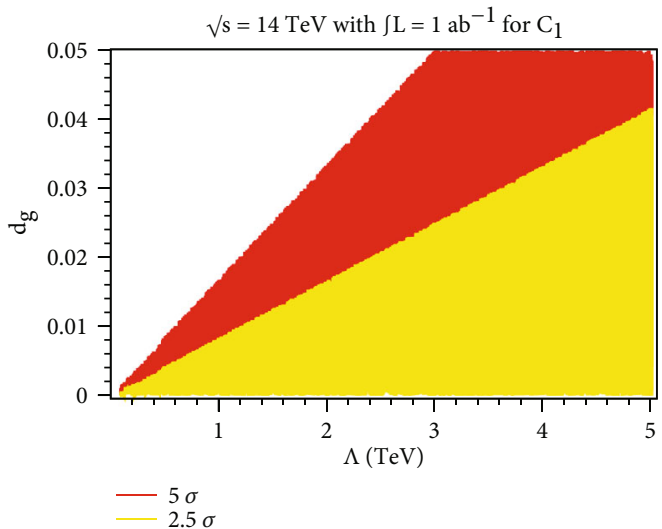

(b)

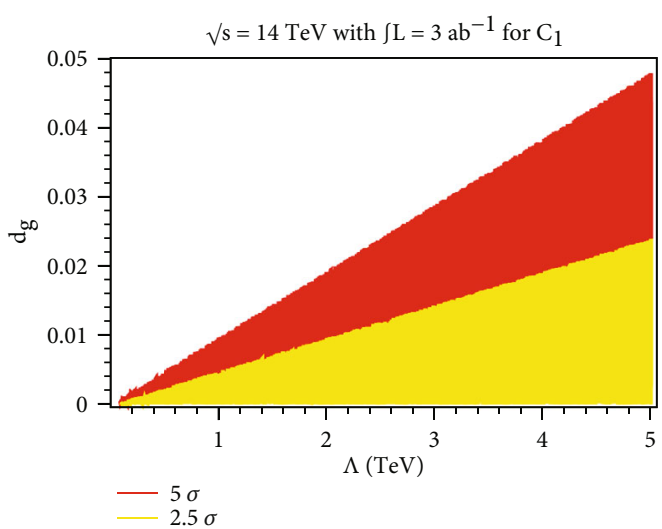

(d)

Figure 6: $d_{g}$ vs. $\Lambda$ for observable $\mathscr{C}_{1}$ at $\sqrt{S}=14 \mathrm{TeV}$ energy at LHC for an integrated luminosity of (a) $0.3 \mathrm{ab}^{-1}$, (b) $1 \mathrm{ab}^{-1}$, (c) $2 \mathrm{ab}^{-1}$, and (d) $3 \mathrm{ab}^{-1}$ respectively.

$\mathscr{C}_{6}$ requires information regarding the beam direction, a lepton having a positive charge and the associated $b$ -quark and identifying a lepton having a negative charge and the associated anti- $b$-quark. Observable $\mathscr{C}_{7}$ requires information of the beam direction and leptons having a positive and negative charge. Similarly observable $\mathscr{C}_{8}$ requires information of the beam direction, $b$-quark and anti- $b$-quark. In the next section, we will discuss the numerical simulation in detail.

\section{Numerical Analysis}

In order to perform our study, we first produced $t \bar{t}$ pairs through the process $p p \longrightarrow t \bar{t}$ and allowed them to decay semileptonically into $\left(b l^{+} v_{l}\right)\left(\bar{b} l^{-} \bar{v}_{l}\right)$ subsequently with the aid of MadGraph5 [42-44] at Leading order (LO) using the decay chain feature described in Ref. [44]. Later these events are interfaced to Pythia8 [45] for Showering Hadronization. The CP-violating interactions discussed in Eqs. (2) and (6) have been incorporated in the MadGraph5 via incorporating the Lagrangian given in Eq. (1) in FeynRules [46]. The events are generated with the following selection criteria:

$$
\begin{gathered}
P_{T}\left(l^{ \pm}\right)>20 \mathrm{GeV}, \\
P_{T}(b, \bar{b})>25 \mathrm{GeV}, \\
\eta\left(b, \bar{b}, l^{ \pm}\right)<2.5, \\
\Delta R(b \bar{b})>0.4, \\
\Delta R\left(l^{+} l^{-}\right)>0.2, \\
\Delta R(b l)>0.4, \\
E_{T}>30 \mathrm{GeV} .
\end{gathered}
$$

The experimental values of the input parameters considered in our study are presented in Table 1, the renormalisation and factorisation scale has been set to $91.188 \mathrm{GeV}$ and the parton distribution functions had been considered to be nn23lo1 [47, 48].

In order to estimate the asymmetries at the LHC, we generate $p p \longrightarrow t \bar{t} \longrightarrow\left(b l^{+} v_{l}\right)\left(\bar{b} l^{-} \bar{v}_{l}\right)$ events with the aid of MadGraph 5 at $13 \mathrm{TeV}$ and $14 \mathrm{TeV}$ LHC energies with distinctive values of coupling constant $\left(d_{g}\right)$ and scale parameter $(\Lambda)$ for the observables given in Eqs. (2) and (6). The values of coupling constant $d_{g}$ and scale parameter $\Lambda$ have been 


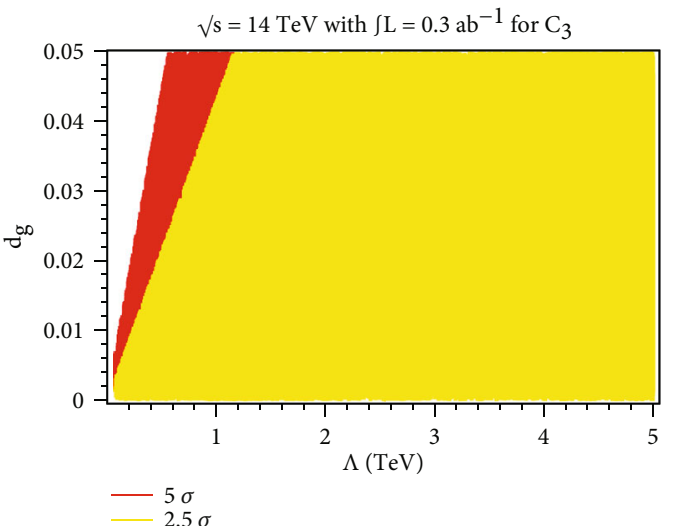

(a)

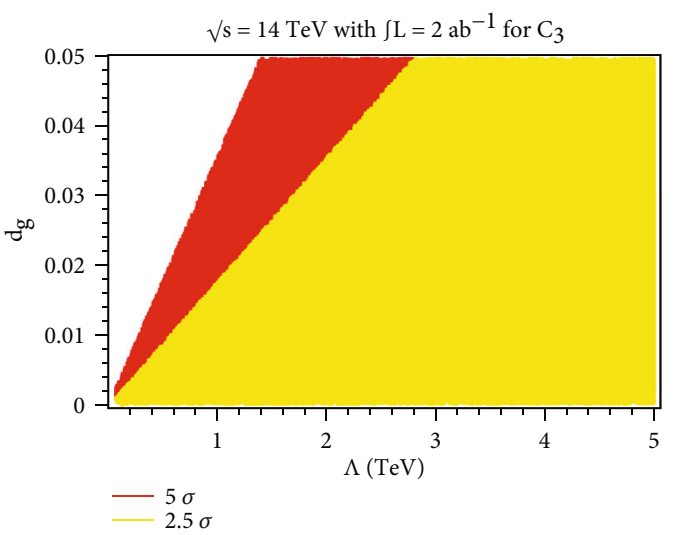

(c)

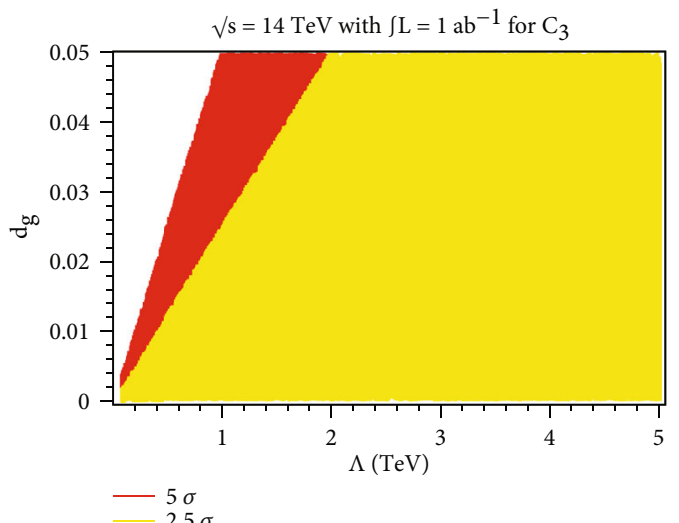

(b)

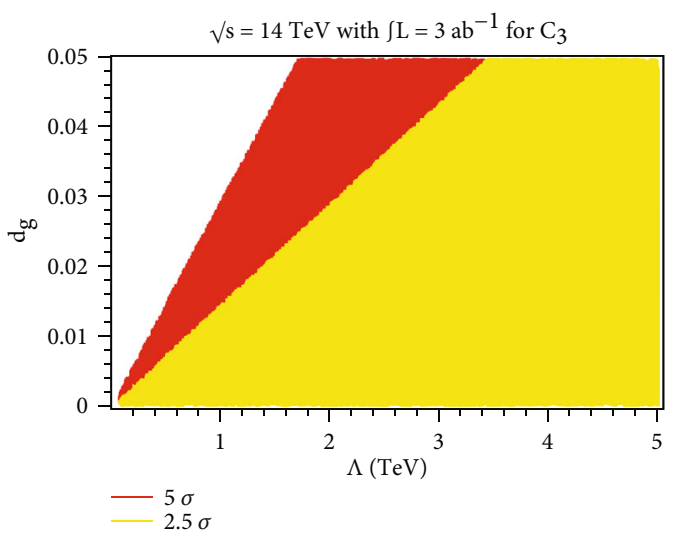

(d)

Figure $7: d_{g}$ vs. $\Lambda$ for observable $\mathscr{C}_{3}$ at $\sqrt{S}=14 \mathrm{TeV}$ energy at LHC for an integrated luminosity of (a) $0.3 \mathrm{ab}^{-1}$, (b) $1 \mathrm{ab}^{-1}$, (c) $2 \mathrm{ab}^{-1}$, and (d) $3 \mathrm{ab}^{-1}$ respectively.

considered from 0 to $5 \times 10^{-2}$ and $M_{W}$ to $2 \mathrm{TeV}$ respectively where $d_{g}=0$ is actually SM. The associated CP-violating asymmetry for the observables listed in Eqs. (2) and (6) is constructed using the formula:

$$
\mathscr{A}_{\mathrm{CP}}=\frac{N\left(\mathscr{C}_{i}>0\right)-N\left(\mathscr{C}_{i}<0\right)}{N\left(\mathscr{C}_{i}>0\right)+N\left(\mathscr{C}_{i}<0\right)},
$$

where the numerator represents the difference between the number of events having positive and negative values of the observable whereas the denominator represents the total number of events. Clearly, for a CP-symmetric observable, $\mathscr{A}_{\mathrm{CP}}$ would be zero because the number of events with a positive value of observable will be equal to the number of events with a negative value of observable and non-zero otherwise. The number of experimentally measured $p p \longrightarrow t \bar{t} \longrightarrow\left(b l^{+}\right.$ $\left.v_{l}\right)\left(\bar{b} l^{-} \bar{v}_{l}\right)$ events at the LHC are given by

$$
N^{\exp }=\sigma^{\exp } \times \operatorname{Br}(t \longrightarrow b l v)^{2} \times\left(b_{\text {tag }}\right)^{2} \times \varepsilon_{\text {eff }} \times \int \mathscr{L} d t
$$

where $\sigma^{\exp }$ represents the experimentally measured value of the $t \bar{t}$ cross-section at a given C.M. energy at the LHC, $b_{\text {tag }}$ is the $b$-tagging efficiency, $\varepsilon_{\text {eff }}$ is the efficiency of cuts and $\int$ $\mathscr{L} d t$ represents the integrated luminosity at the LHC. The sensitivity for a given observable could be estimated by comparing the $\mathscr{A}_{\mathrm{CP}}$ corresponding to the underlying observable with the following experimental sensitivity at a given confidence level (C.L.) $n_{c l}$ :

$$
\mathscr{A}^{\exp }=\frac{n_{c l}}{\sqrt{N^{\exp }}}
$$

These are discussed in Figures 2-9 for $\sqrt{S}=13 \mathrm{TeV}$ and $14 \mathrm{TeV}$ at the LHC. The values of asymmetries corresponding to various CP-violating observables discussed in Eqs. (2) and (6) are also presented for various values of $\Lambda$ and $d_{g}$. We estimate asymmetries for $d_{g}$ from 0 to 0.05 and $\Lambda$ between $M_{W}$ to $2 \mathrm{TeV}$ for $\sqrt{S}=13 \mathrm{TeV}$ and $14 \mathrm{TeV}$ at the LHC. In Tables 2 and 3, we present asymmetries corresponding to various observables at $\sqrt{S}=13 \mathrm{TeV}$ and $14 \mathrm{TeV}$ LHC energies. From these tables, it is clear that the asymmetries corresponding to observables $C_{2}, C_{6}, C_{7}$, and $C_{8}$ are within the limits of statistical uncertainties and therefore would not be useful to calculate CP-violation sensitivity as these are consistent with SM. However, asymmetries related to observables $\mathscr{C}_{1}, \mathscr{C}_{3}, \mathscr{C}_{4}$, and $\mathscr{C}_{5}$ are found to be non-zero at $3 \sigma$ C.L.. It is, therefore, informative to discuss the asymmetries obtained for observables $\mathscr{C}_{1}, \mathscr{C}_{3}, \mathscr{C}_{4}$, and $\mathscr{C}_{5}$ in detail as these are expected to be more sensitive. 


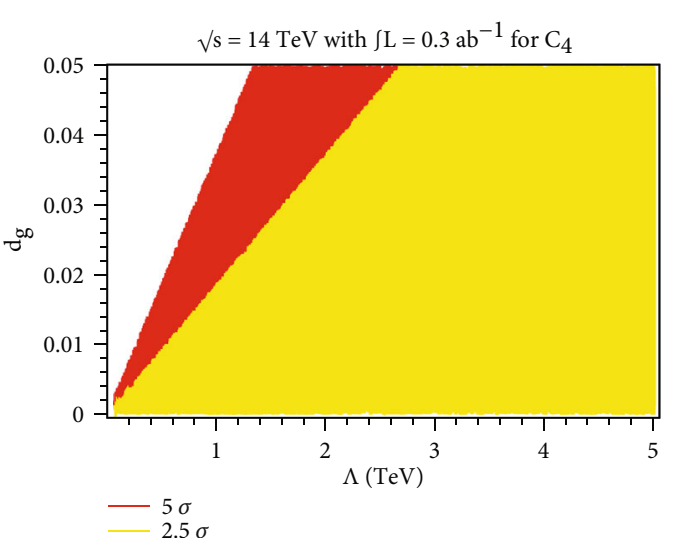

(a)

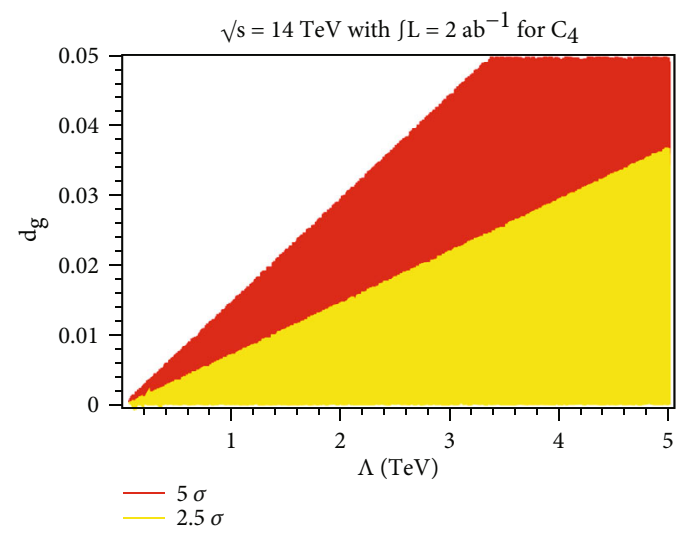

(c)

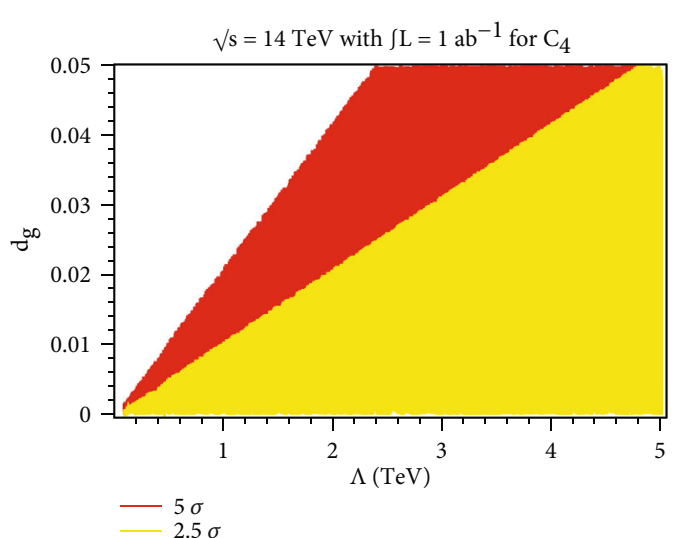

(b)

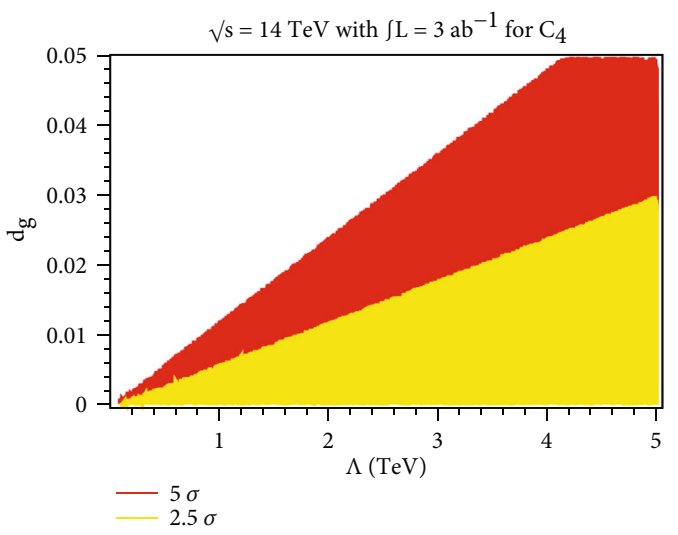

(d)

Figure 8: $d_{g}$ vs. $\Lambda$ for observable $\mathscr{C}_{4}$ at $\sqrt{S}=14 \mathrm{TeV}$ energy at LHC for an integrated luminosity of (a) $0.3 \mathrm{ab}^{-1}$, (b) $1 \mathrm{ab}^{-1}$, (c) $2 \mathrm{ab}^{-1}$, and (d) $3 \mathrm{ab}^{-1}$ respectively.

From Tables 2 and 3, it is also clear that if we fix the CPviolating scale to a certain value the asymmetries increase linearly with $d_{g}$ which supports the results in Refs. [28, 41]. Conversely, limiting the coupling $d_{g}$ to a constant value and increasing the value of $\Lambda$ reduces the value of the resulting asymmetries. This suggests that large $\mathrm{CP}$-violation sensitivity can be achieved in two ways, either increasing $d_{g}$ or decreasing $\Lambda$. Furthermore, the asymmetries obtained at the $\sqrt{S}=14 \mathrm{TeV}$ energy at LHC, presented in Table 3, show similar results as observed for the $13 \mathrm{TeV}$ LHC energy. According to the above tables, we infer that the largest asymmetry corresponds to the observable $\mathscr{C}_{1}$. The results corresponding to non-zero asymmetry could also be summarized as

$$
\begin{aligned}
& \mathscr{A}_{1}=0.0023+119.3 \frac{d_{g}}{\Lambda}, \\
& \mathscr{A}_{3}=0.0007+39.9 \frac{d_{g}}{\Lambda}, \\
& \mathscr{A}_{4}=-0.0018-96.1 \frac{d_{g}}{\Lambda}, \\
& \mathscr{A}_{5}=0.0018+93.6 \frac{d_{g}}{\Lambda},
\end{aligned}
$$

respectively for observables $\mathscr{C}_{1}, \mathscr{C}_{3}, \mathscr{C}_{4}$, and $\mathscr{C}_{5}$.
It is to be noted that for estimating the experimental uncertainties in event rates we first combined the ATLAS [50] and CMS [51] experimental uncertainties observed with 2015 and 2016 data during LHC Run II for the top pair at $\sqrt{S}=13 \mathrm{TeV}$ for $36.1 \mathrm{fb}^{-1}$ presented in Ref. [52]. In order to calculate experimental sensitivity, we first combined the ATLAS and CMS cross-sections which are as follows:

$$
\begin{aligned}
\sigma^{\text {ATLAS }} & =803 \pm 7(\text { stat }) \pm 27(\text { Syst }) \pm 45(\text { lumi }) \pm 12(\text { beam }) \mathrm{pb} \\
\sigma^{\mathrm{CMS}} & =793 \pm 8(\text { stat }) \pm 38(\text { Syst }) \pm 21(\text { lumi }) \mathrm{pb}, \\
\sigma^{\mathrm{LHC}} & =798 \pm 49.25 \mathrm{pb} .
\end{aligned}
$$

Event rates were then estimated by combining the crosssection with the luminosity, branching ratios for the $t \longrightarrow b$ $l v_{l}$ and the $b$-tagging efficiency which is assumed to be $56 \%$. A similar calculation has been performed for $\sqrt{S}=14 \mathrm{TeV}$ with a theoretical cross-section $953.6_{-33.9-17.8}^{+22.7+16.2} \mathrm{pb}$ at the NNLO+NNLL level [53] for the projected integrated luminosities of the LHC of $\int \mathscr{L} d t=0.3 \mathrm{ab}^{-1}, 1 \mathrm{ab}^{-1}, 2 \mathrm{ab}^{-1}$, and $3 \mathrm{ab}^{-1}$. We show the results for $13 \mathrm{TeV}$ and $14 \mathrm{TeV}$ C.M. energies at LHC for $d_{g}$ vs. $\Lambda$ at various confidence levels in Figures 2-9. We present the results for $\Lambda$ between the range 


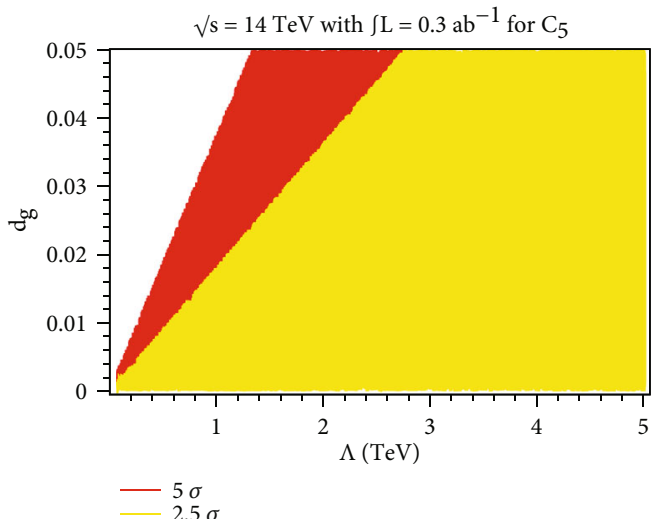

(a)

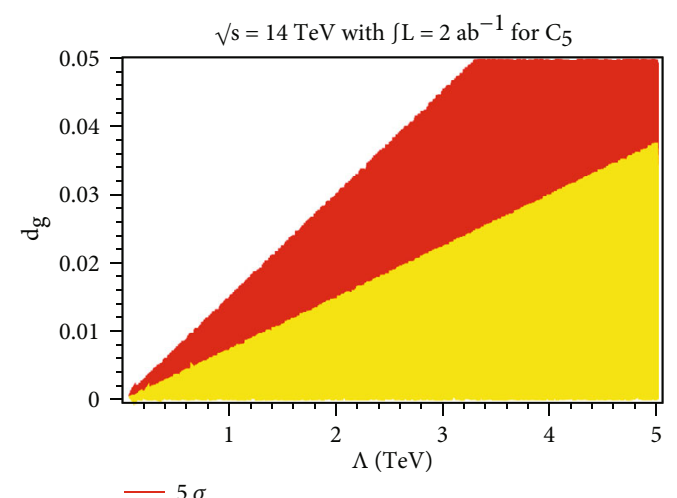

$-5 \sigma$

(c)

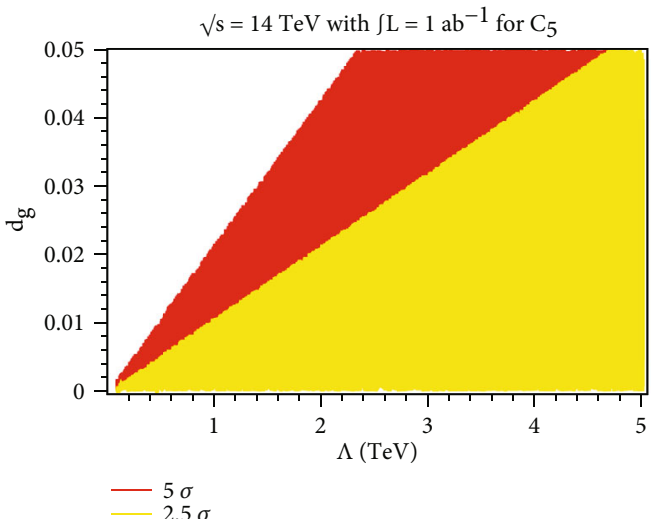

(b)

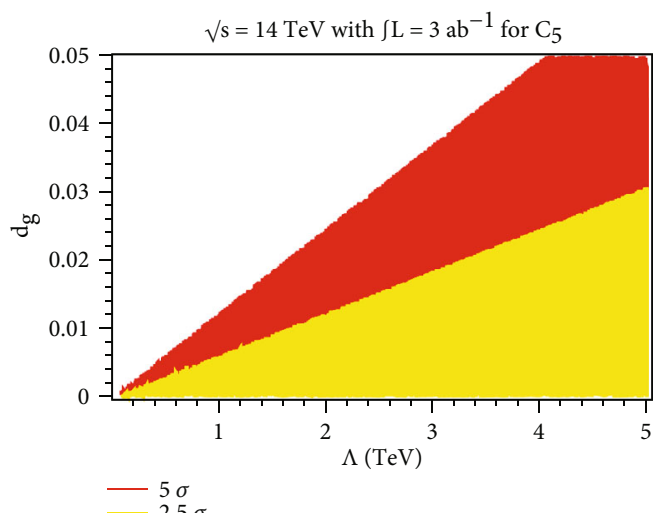

(d)

Figure 9: $d_{g}$ vs. $\Lambda$ for observable $\mathscr{C}_{5}$ at $\sqrt{S}=14 \mathrm{TeV}$ energy at LHC for an integrated luminosity of (a) $0.3 \mathrm{ab}^{-1}$, (b) $1 \mathrm{ab}^{-1}$, (c) $2 \mathrm{ab}^{-1}$, and (d) $3 \mathrm{ab}^{-1}$ respectively.

TABLE 2: Integrated asymmetries (in \%) at LO for $\sqrt{S}=13 \mathrm{TeV}$ at LHC for the process $p p \longrightarrow t \bar{t} \longrightarrow\left(b l^{+} v_{l}\right)\left(\bar{b} l^{-} \bar{v}_{l}\right)$ corresponding to various observables for distinct choices of $d_{g}$ and $\Lambda$. The statistical uncertainty at the $1 \sigma$ confidence level in all the results is estimated to be about $3 \times 10^{-4}$.

\begin{tabular}{|c|c|c|c|c|c|c|c|c|c|}
\hline$\Lambda$ & $\begin{array}{c}d_{g} \\
\mathrm{SM}\end{array}$ & $\begin{array}{c}\mathscr{A}_{1} \\
0.05\end{array}$ & $\begin{array}{c}\mathscr{A}_{2} \\
0.01\end{array}$ & $\begin{array}{c}\mathscr{A}_{3} \\
0.02\end{array}$ & $\begin{array}{c}\mathscr{A}_{4} \\
-0.05\end{array}$ & $\begin{array}{c}\mathscr{A}_{5} \\
0.03\end{array}$ & $\begin{array}{c}\mathscr{A}_{6} \\
0.05\end{array}$ & $\begin{array}{c}\mathscr{A}_{7} \\
-0.05\end{array}$ & $\begin{array}{c}\mathscr{A}_{8} \\
0.04\end{array}$ \\
\hline \multirow{3}{*}{$\mathrm{M}_{W}$} & $5 \times 10^{-3}$ & 1.17 & 0 & 0.37 & -0.91 & 0.89 & 0.02 & -0.01 & -0.01 \\
\hline & $1 \times 10^{-2}$ & 2.22 & 0 & 0.72 & -1.74 & 1.74 & 0.04 & -0.02 & 0.04 \\
\hline & $5 \times 10^{-2}$ & 6.29 & 0.02 & 2.12 & -5.11 & 4.98 & 0.01 & -0.01 & 0.03 \\
\hline \multirow{3}{*}{$0.5 \mathrm{TeV}$} & $5 \times 10^{-3}$ & 0.19 & 0.01 & 0.02 & -0.15 & 0.13 & -0.01 & 0.03 & -0.03 \\
\hline & $1 \times 10^{-2}$ & 0.38 & 0.04 & 0.17 & -0.29 & 0.31 & -0.03 & 0.05 & -0.06 \\
\hline & $5 \times 10^{-2}$ & 1.79 & -0.01 & 0.56 & -1.47 & 1.37 & -0.02 & 0.03 & -0.04 \\
\hline \multirow{3}{*}{$1 \mathrm{TeV}$} & $5 \times 10^{-3}$ & 0.06 & 0.02 & 0.07 & -0.11 & 0.04 & -0.03 & 0.03 & -0.09 \\
\hline & $1 \times 10^{-2}$ & 0.14 & -0.03 & 0.08 & -0.12 & 0.13 & -0.04 & 0.02 & 0 \\
\hline & $5 \times 10^{-2}$ & 0.92 & 0 & 0.28 & -0.72 & 0.74 & -0.03 & 0 & 0.03 \\
\hline \multirow{3}{*}{$2 \mathrm{TeV}$} & $5 \times 10^{-3}$ & 0.03 & -0.01 & -0.01 & -0.01 & 0.04 & 0.01 & 0 & 0 \\
\hline & $1 \times 10^{-2}$ & 0.12 & 0.02 & 0.03 & -0.10 & 0.10 & 0 & 0.01 & -0.03 \\
\hline & $5 \times 10^{-2}$ & 0.42 & 0.02 & 0.13 & -0.33 & 0.32 & 0.02 & -0.03 & -0.04 \\
\hline
\end{tabular}


TABLE 3: Integrated asymmetries (in \%) at LO for $\sqrt{S}=14 \mathrm{TeV}$ at LHC for the process $p p \longrightarrow t \bar{t} \longrightarrow\left(b l^{+} v_{l}\right)\left(\bar{b} l^{-} \bar{v}_{l}\right)$ corresponding to various observables for distinct choices of $d_{g}$ and $\Lambda$. The statistical uncertainty at the $1 \sigma$ confidence level in all the results is estimated to be about $3 \times 10^{-4}$.

\begin{tabular}{|c|c|c|c|c|c|c|c|c|c|}
\hline$\Lambda$ & $\begin{array}{l}d_{g} \\
\mathrm{SM}\end{array}$ & $\begin{array}{c}\mathscr{A}_{1} \\
-0.03\end{array}$ & $\begin{array}{c}\mathscr{A}_{2} \\
0.02\end{array}$ & $\begin{array}{c}\mathscr{A}_{3} \\
0.01\end{array}$ & $\begin{array}{c}\mathscr{A}_{4} \\
0.07\end{array}$ & $\begin{array}{c}\mathscr{A}_{5} \\
-0.04\end{array}$ & $\begin{array}{c}\mathscr{A}_{6} \\
-0.03\end{array}$ & $\begin{array}{c}\mathscr{A}_{7} \\
-0.03\end{array}$ & $\begin{array}{c}\mathscr{A}_{8} \\
-0.03\end{array}$ \\
\hline \multirow{3}{*}{$\mathrm{M}_{W}$} & $5 \times 10^{-3}$ & 1.12 & 0 & 0.36 & -0.95 & 0.88 & -0.03 & 0.01 & -0.01 \\
\hline & $1 \times 10^{-2}$ & 2.24 & 0.03 & 0.70 & -1.73 & 1.74 & -0.03 & -0.04 & -0.01 \\
\hline & $5 \times 10^{-2}$ & 6.39 & -0.02 & 2.09 & -5.11 & 4.97 & 0.05 & -0.01 & -0.03 \\
\hline \multirow{3}{*}{$0.5 \mathrm{TeV}$} & $5 \times 10^{-3}$ & 0.17 & -0.04 & 0.10 & -0.13 & 0.14 & 0.02 & 0.03 & -0.04 \\
\hline & $1 \times 10^{-2}$ & 0.36 & 0.01 & 0.14 & -0.33 & 0.24 & 0.01 & 0.01 & -0.03 \\
\hline & $5 \times 10^{-2}$ & 1.89 & -0.06 & 0.62 & -1.46 & 1.46 & 0.02 & 0.07 & -0.03 \\
\hline \multirow{3}{*}{$1 \mathrm{TeV}$} & $5 \times 10^{-3}$ & 0.14 & 0.03 & 0.05 & -0.10 & 0.12 & 0.02 & -0.04 & -0.01 \\
\hline & $1 \times 10^{-2}$ & 0.21 & 0.03 & 0.02 & -0.21 & 0.16 & -0.03 & 0.02 & 0 \\
\hline & $5 \times 10^{-2}$ & 0.89 & 0.01 & 0.23 & -0.75 & 0.75 & 0 & 0.03 & -0.06 \\
\hline \multirow{3}{*}{$2 \mathrm{TeV}$} & $5 \times 10^{-3}$ & 0.07 & 0.01 & 0.03 & -0.03 & 0.09 & -0.03 & -0.05 & -0.01 \\
\hline & $1 \times 10^{-2}$ & 0.09 & -0.02 & 0.07 & -0.04 & 0.05 & -0.01 & 0 & 0 \\
\hline & $5 \times 10^{-2}$ & 0.45 & -0.03 & 0.14 & -0.33 & 0.33 & 0 & -0.01 & 0.03 \\
\hline
\end{tabular}

0 to $5 \mathrm{TeV}$ but we had actually performed the study in the range $M_{W}$ to $2 \mathrm{TeV}$.

In Figures 2-9 the area shown in white is discarded by restricting the contribution in top-pair cross-section to be consistent with the SM within $3 \sigma$ statistical errors whereas the yellow and red regions show possible $d_{g}-\Lambda$ space allowed at $2.5 \sigma$ and $5 \sigma$ respectively for the given C.M. energy and Luminosities. We have a wide range of $\tilde{d}_{g}\left(d_{g} / \Lambda\right)$ values at which we can observe $5 \sigma$ sensitivity at $13 \mathrm{TeV}$ and $14 \mathrm{TeV}$ LHC energies. From the figures, we can get a rough estimate of minimum bound on $d_{g}$ and $\Lambda$ and can find the lower limit on $\tilde{d}_{g}\left(d_{g} / \Lambda\right)$ at $5 \sigma$ C.L..

Finally, we calculate the exact limits on $\tilde{d}_{g}$ corresponding to the most promising observable $\mathscr{C}_{1}$ at $\sqrt{S}=13 \mathrm{TeV}$ and $14 \mathrm{TeV}$ energy at LHC. The experimental sensitivity at $\sqrt{S}$ $=13 \mathrm{TeV}$ energy at LHC is found to be $0.2 \%$ at $1 \sigma$ C.L. and the similar value at $5 \sigma$ C.L. would be $1.0 \%$. This translates into the values of $\left|d_{g} / \Lambda\right|$ of about $\gtrsim 0.6 \times 10^{-4} \mathrm{GeV}^{-1}, 0.2 \times$ $10^{-4}$ at $5 \sigma$ C.L. at $13 \mathrm{TeV}$ C.M. energy with the integrated luminosities of $36.1 \mathrm{fb}^{-1}, 140 \mathrm{fb}^{-1}$ respectively for observable $\mathscr{C}_{1}$. Similarly, at $14 \mathrm{TeV}$ C.M. energy at LHC the value of $\mid d_{g}$ $/ \Lambda \mid$ should be $z 0.6 \times 10^{-5} \mathrm{GeV}^{-1}, 0.5 \times 10^{-5} \mathrm{GeV}^{-1}, 0.9 \times 1$ $0^{-5} \mathrm{GeV}^{-1}$, and $0.1 \times 10^{-4} \mathrm{GeV}^{-1}$ at $5 \sigma$ C.L. for the projected luminosities of $0.3 \mathrm{ab}^{-1}, 1 \mathrm{ab}^{-1}, 2 \mathrm{ab}^{-1}$, and $3 \mathrm{ab}^{-1}$, respectively. The asymmetries $\left(\mathscr{A}_{i}\right)$ corresponding to observables $\left(\mathscr{C}_{i}\right)$ could also be written as

$$
\mathscr{A}_{i}=\mathscr{A}_{\mathrm{SM}}+b_{i} \frac{d_{g}}{\Lambda},
$$

where $b_{i}$ is defined via

$$
b_{i}=\frac{d \mathscr{A}_{i}}{d\left(d_{g} / \Lambda\right)} .
$$

Figure 10 clearly show that asymmetries are almost zero up to $10^{-3}$ and then start increasing slowly. It shows that at large $d_{g} / \Lambda$, sensitivities become quite significant.

The aim of this article is to set bounds on anomalous CPviolating coupling for a situation when the effects due to such interactions are not visible by just event count, rather these could be probed through the observables considered in our study. We have presented $5 \sigma$ sensitivities for $13 \mathrm{TeV}$ C.M. energy at LHC with the integrated luminosities of $36.1 \mathrm{fb}^{-1}$, $140 \mathrm{fb}^{-1}$ for $\sim 19 \mathrm{k}, 73.5 \mathrm{k}$ events per month respectively and predicted that we can achieve $5 \sigma$ sensitivity at $14 \mathrm{TeV}$ LHC energy with projected luminosities of $0.3 \mathrm{ab}^{-1}, 1 \mathrm{ab}^{-1}$, $2 \mathrm{ab}^{-1}$, and $3 \mathrm{ab}^{-1}$ with $\sim 183 \mathrm{k}, 608 \mathrm{k}, 1.2 \mathrm{M}$, and $1.8 \mathrm{M}$ events respectively. The results obtained in our study are based only on statistical uncertainties, systematic uncertainties have not been accounted for. However since it will affect the numerator and denominator in the asymmetry almost equally and therefore it is expected that our results will remain practically unaffected due to the systematic uncertainties. The above finding is also confirmed by earlier studies on such CP asymmetries $[22,54,55]$. Also in a similar manner, although we had performed our analysis at the leading order, the $K$-factor due to higher-order QCD corrections will affect the denominators and numerators of all the asymmetries almost equally and will be therefore canceled and hence the asymmetries will remain unchanged against such corrections. It is important to note that our study differs from the earlier studies by taking into account full matrix-element-squared calculation for $p p \longrightarrow t$ $\bar{t} \longrightarrow\left(b l^{+} v_{l}\right)\left(\bar{b} l^{-} \bar{v}_{l}\right)$ with $l$ being $e$ and $\mu$. In order to probe 

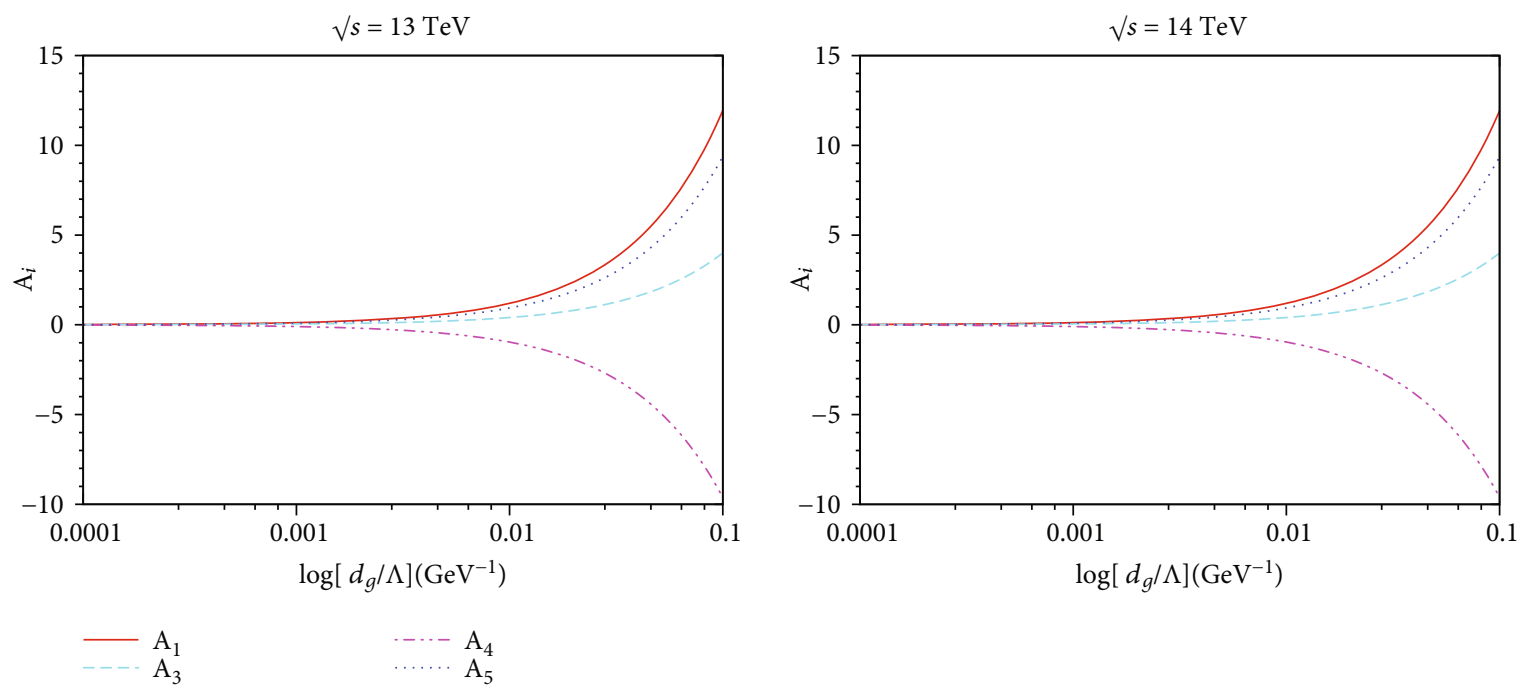

Figure 10: Asymmetries vs. $d_{g} / \Lambda$ for $\sqrt{S}=13 \mathrm{TeV}$ and $14 \mathrm{TeV}$ energy at LHC.

the effects of such interactions the earlier studies only considered the leading effects which are linear in nature. Also, we calculated the counting asymmetries in dilepton channel and used $d_{g}$ and $\Lambda$ as free parameters.

We now compare our results with other relevant works. According to Ref. [41], $5 \sigma$ sensitivity of $\left|\tilde{d}_{g}\right|<0.3 \times 10^{-3} \mathrm{Ge}$ $\mathrm{V}^{-1}$ requires $10 \mathrm{fb}^{-1}$ of data at $14 \mathrm{TeV}$ LHC energy. The corresponding estimates are found to be $-0.8 \times 10^{-4}<\tilde{d}_{g}<0.8$ $\times 10^{-4}$ for the top-quark pair production in association with two photons [56] for an integrated luminosity of $3 \mathrm{ab}^{-1}$ and of about $10^{-4} \mathrm{GeV}^{-1}$ in the context of $e^{-} e^{+}$collider with a data of about $50 \mathrm{fb}^{-1}$ [57]. The indirect limits from the EDM measurements are found to be somewhat stringent, e.g. Ref. [58] reports that $\left|\tilde{d}_{g}\right|<1.1 \times 10^{-5} \mathrm{GeV}^{-1}$ at $90 \%$ C.L. from the measurement of the neutron electric dipole moment.

\section{Summary}

We have analysed the effect of T-odd anomalous couplings of the top-quark with gluons via the top-quark pair production through their semileptonic decay modes at the LHC for $\sqrt{S}$ $=13 \mathrm{TeV}$ and $14 \mathrm{TeV}$ using the T-odd observables discussed in Eqs. (2) and (6). These observables are interesting as these do not require full reconstruction of the $t \bar{t}$, rather these require the momenta of the visible final state particles which in our case are $b l^{+}$and $\bar{b} l^{-}$pairs emerging due to decay of a top and anti-top quarks respectively. The asymmetries corresponding to the T-odd observables have been estimated using Eq. (8) and are presented in Figures 2-9 for $13 \mathrm{TeV}$ and $14 \mathrm{TeV}$ LHC energies. Using the largest asymmetry, $\mathscr{A}_{1}$ which corresponds to the observable $\mathscr{C}_{1}$, we estimated the sensitivity to the CP-violating couplings for $\sqrt{S}=13 \mathrm{TeV}$ energy at LHC with the integrated luminosities of $\int \mathscr{L} d t=$ $36.1 \mathrm{fb}^{-1}, 140 \mathrm{fb}^{-1}$ to be $\left|d_{g} / \Lambda\right| \leqslant 0.29 \times 10^{-4} \mathrm{GeV}^{-1}, 0.52 \times$ $10^{-5} \mathrm{GeV}^{-1}$ at $3 \sigma$ C.L. and $\left|d_{g} / \Lambda\right| \leqslant 0.6 \times 10^{-4} \mathrm{GeV}^{-1}, 0.2 \times$ $10^{-4} \mathrm{GeV}^{-1}$ at $5 \sigma$ C.L. respectively. The corresponding esti-
TABLE 4: Sensitivity to CP-violating anomalous couplings at $3 \sigma$ C.L. and $5 \sigma$ C.L. in the process $p p \longrightarrow t \bar{t} \longrightarrow\left(b l^{+} v_{l}\right)\left(\bar{b} l^{-} \bar{v}_{l}\right)$ at $\sqrt{S}=13$ $\mathrm{TeV}$ energy at LHC with the integrated luminosities of $36.1 \mathrm{fb}^{-1}$ and $140 \mathrm{fb}^{-1}$ and HL-LHC with $\sqrt{S}=14 \mathrm{TeV}$ energy with the projected luminosities of $0.3 \mathrm{ab}^{-1}, 1 \mathrm{ab}^{-1}, 2 \mathrm{ab}^{-1}$, and $3 \mathrm{ab}^{-1}$.

\begin{tabular}{llll}
\hline \multirow{2}{*}{$S(\mathrm{TeV})$} & \multirow{2}{*}{$\mathscr{L}_{t}$} & \multicolumn{2}{c}{$\left|d_{g} / \Lambda\right|\left(\mathrm{in} \mathrm{GeV}^{-1}\right)$} \\
& & At $3 \sigma \mathrm{C} . \mathrm{L}$. & At $5 \sigma$ C.L. \\
\hline \multirow{2}{*}{13} & $36.1 \mathrm{fb}^{-1}$ & $0.29 \times 10^{-4}$ & $0.6 \times 10^{-4}$ \\
& $140 \mathrm{fb}^{-1}$ & $0.52 \times 10^{-5}$ & $0.2 \times 10^{-4}$ \\
\hline \multirow{3}{*}{$14(\mathrm{HL}-\mathrm{LHC})$} & $0.3 \mathrm{ab}^{-1}$ & $0.39 \times 10^{-5}$ & $0.6 \times 10^{-5}$ \\
& $1.0 \mathrm{ab}^{-1}$ & $0.11 \times 10^{-4}$ & $0.5 \times 10^{-5}$ \\
& $2.0 \mathrm{ab}^{-1}$ & $0.13 \times 10^{-4}$ & $0.9 \times 10^{-5}$ \\
& $3.0 \mathrm{ab}^{-1}$ & $0.14 \times 10^{-4}$ & $0.1 \times 10^{-4}$ \\
\hline
\end{tabular}

mates for the HL-LHC with $\sqrt{S}=14 \mathrm{TeV}$ and $\int \mathscr{L} d t=0.3 \mathrm{a}$ $\mathrm{b}^{-1}, 1 \mathrm{ab}^{-1}, 2 \mathrm{ab}^{-1}$, and $3 \mathrm{ab}^{-1}$ would yield $\left|d_{g} / \Lambda\right| \lesssim 0.39 \times 1$ $0^{-5} \mathrm{GeV}^{-1}, 0.11 \times 10^{-4} \mathrm{GeV}^{-1}, 0.13 \times 10^{-4} \mathrm{GeV}^{-1}$, and 0.14 $\times 10^{-4} \mathrm{GeV}^{-1}$ at $3 \sigma$ C.L. and $\left|d_{g} / \Lambda\right| \leqslant 0.6 \times 10^{-5} \mathrm{GeV}^{-1}, 0.5$ $\times 10^{-5} \mathrm{GeV}^{-1}, 0.9 \times 10^{-5} \mathrm{GeV}^{-1}$, and $0.1 \times 10^{-4} \mathrm{GeV}^{-1}$ at 5 $\sigma$ C.L. respectively. These results have been summarised in Table 4 and seem to be setting stringent bounds on the CPviolating couplings of the top-quark and therefore a detailed experimental investigation is worthwhile to shed light on such CP-violating couplings of the top-quark.

\section{Data Availability}

The data used to support the findings of this study are included within the article.

\section{Conflicts of Interest}

The authors declare that they have no conflicts of interest. 


\section{Acknowledgments}

This work was supported in part by University Grant Commission under a Start-Up Grant no. F30-377/2017 (BSR). We thank Ravindra Yadav for his assistance regarding high-performance computing, Manjari Sharma and Surabhi Gupta for some valuable discussions. We acknowledge the use of cluster computing facility at the ReCAPP, HRI, Allahabad, India, during the initial phase of the work.

\section{References}

[1] J. H. Christenson, J. W. Cronin, V. L. Fitch, and R. Turlay, "Evidence for the $2 \pi$ decay of the $K_{2}^{0}$ Meson," Physical Review Letters, vol. 13, no. 4, pp. 138-140, 1964.

[2] E. J. Chun, G. Cvetič, P. S. B. Dev et al., "Probing leptogenesis," International Journal of Modern Physics A, vol. 33, article 1842005, 2018.

[3] S. Antusch, E. Cazzato, M. Drewes et al., "Probing leptogenesis at future colliders," Journal of High Energy Physics, vol. 2018, article 124, no. 9, 2018.

[4] A. Achelashvili and Z. Tavartkiladze, "Leptonic CP violation and leptogenesis," in , Article ID 020012AIP Conference Proceedings, vol. 1900, Lead/Deadwood, SD, United States, 2017.

[5] K. Moffat, S. Pascoli, S. T. Petcov, and J. Turner, "Leptogenesis from low energy CP violation," Journal of High Energy Physics, vol. 2019, no. 3, article 34, 2019.

[6] Y. Cui and B. Shuve, "Probing baryogenesis with displaced vertices at the LHC," Journal of High Energy Physics, vol. 2015, no. 2, article 49, 2015.

[7] G. Barbiellini, G. Bonneaud, G. Coignet et al., "The production and detection of Higgs particles at LEP.

[8] A. I. Vainshtein, V. I. Zakharov, and M. A. Shifman, "Higgs particles," Soviet Physics Uspekhi, vol. 23, no. 8, pp. 429-449, 1980.

[9] G. G. Raffelt, "Dark matter: motivation, candidates and searches,” https://arxiv.org/abs/hep-ph/9712538.

[10] F. Halzen, T. Stelzer, and M. Kamionkowski, "Signatures of dark matter in underground detectors," Physical Review D, vol. 45, no. 12, pp. 4439-4442, 1992.

[11] M. Kamionkowski, "Possible relics from new physics in the early universe: inflation, the cosmic microwave background, and particle dark matter," https://arxiv.org/abs/astro-ph/ 9809214

[12] P. Krawczyk, D. London, R. D. Peccei, and H. Steger, "Predictions of the CKM model for CP asymmetries in B decay," Nuclear Physics B, vol. 307, no. 1, pp. 19-33, 1988.

[13] T. Bhattacharya, B. Yoon, R. Gupta, and V. Cirigliano, "Neutron electric dipole moment from beyond the standard model," https://arxiv.org/abs/1812.06233.

[14] E. Mereghetti, "Electric dipole moments: a theory overview," https://arxiv.org/abs/1810.01320.

[15] W. Bernreuther, L. Chen, I. García et al., "CP-violating top quark couplings at future linear $e^{+} e^{-}$colliders," The European Physical Journal C, vol. 78, no. 2, p. 78, 2018.

[16] K. Hagiwara, H. Yokoya, and Y. J. Zheng, "Probing the CP properties of top Yukawa coupling at an $e^{+} e^{-}$collider," Journal of High Energy Physics, vol. 2018, article 180, no. 2, 2018.

[17] The ATLAS collaboration, M. Aaboud, G. Aad et al., "Measurements of charge and CP asymmetries in $b$-hadron decays using top-quark events collected by the ATLAS detector in $p$ p collisions at $\sqrt{s}=8 \mathrm{TeV}$," Journal of High Energy Physics, vol. 2017, no. 2, 2017.

[18] A. Hayreter and G. Valencia, "T-odd correlations from the top-quark chromoelectric dipole moment in lepton plus jets top-pair events," Physical Review D, vol. 93, no. 1, article 014020, 2016.

[19] P. Saha, K. Kiers, B. Bhattacharya, D. London, A. Szynkman, and J. Melendez, "MeasuringCP-violating observables in rare top decays at the LHC," Physical Review D, vol. 93, no. 5, article 054044, 2016.

[20] S. Berge, M. Mühlleitner, A. Moreno Briceño, D. Wackeroth, and M. Wiebusch, "CP-violation in top quark pair production in the complex MSSM at hadron colliders," Nuclear and Particle Physics Proceedings, vol. 267-269, pp. 294-301, 2015.

[21] G. Aad and et al.ATLAS Collaboration, "ATLAS-CONF-2013$032 "$.

[22] J. de Vries, P. Draper, K. Fuyuto, J. Kozaczuk, and D. Sutherland, "Indirect signs of the Peccei-Quinn mechanism," Physical Review D, vol. 99, no. 1, article 015042, 2019.

[23] V. Cirigliano, W. Dekens, J. de Vries, and E. Mereghetti, "Constraining the top-Higgs sector of the standard model effective field theory," Physical Review D, vol. 94, no. 3, article 034031, 2016.

[24] Y. T. Chien, V. Cirigliano, W. Dekens, J. de Vries, and E. Mereghetti, "Direct and indirect constraints on $\mathrm{CP}$ violating Higgs-quark and Higgs-gluon interactions," Journal of High Energy Physics, vol. 2016, no. 2, article 11, 2016.

[25] K. Cheung, "Probing nonstandard top-quark couplings using spin correlation," Physical Review D, vol. 55, no. 7, pp. 44304434, 1997.

[26] K. M. Cheung, "Probing the chromoelectric and chromomagnetic dipole moments of the top quark at hadronic colliders," Physical Review D, vol. 53, no. 7, pp. 3604-3615, 1996.

[27] H. T. Li and G. Valencia, " $C P$ violating anomalous couplings in $W$ jet production at the LHC," Physical Review D, vol. 96, no. 7, article 075014, 2017.

[28] S. K. Gupta and G. Valencia, " $C P$-odd correlations using jet momenta from $t \bar{t}$ events at the Tevatron," Physical Review D, vol. 81, no. 3, article 034013, 2010.

[29] O. Antipin and G. Valencia, " $T$-odd correlations from $C P$ violating anomalous top-quark couplings revisited," Physical Review D, vol. 79, no. 1, article 013013, 2009.

[30] The CMS collaboration, V. Khachatryan, A. M. Sirunyan et al., "Search for CP violation in $t \bar{t}$ production and decay in protonproton collisions at $\sqrt{s}=8 \mathrm{TeV}$," Journal of High Energy Physics, vol. 2017, no. 3, 2017.

[31] S. Taheri Monfared, S. Fayazbakhsh, and M. Mohammadi Najafabadi, "Exploring anomalous $H Z \gamma$ couplings in $\gamma$-proton collisions at the LHC," Physics Letters B, vol. 762, no. 762, pp. 301-308, 2016.

[32] S. Dawson, S. K. Gupta, and G. Valencia, " $C P$ violating anomalous couplings in $W \gamma$ and $Z \gamma$ production at the LHC," Physical Review D, vol. 88, no. 3, article 035008, 2013.

[33] S. Dwivedi, D. K. Ghosh, B. Mukhopadhyaya, and A. Shivaji, "Distinguishing CP-odd couplings of the Higgs boson to weak boson pairs," Physical Review D, vol. 93, no. 11, article 115039, 2016.

[34] W. Bernreuther and Z. G. Si, "Distributions and correlations for top quark pair production and decay at the Tevatron and LHC," Nuclear Physics B, vol. 837, no. 1-2, pp. 90-121, 2010. 
[35] P. Poulose and S. D. Rindani, " $C P$-violating $Z \gamma \gamma$ and topquark electric dipole couplings in $\gamma \gamma \longrightarrow t t$," Physics Letters $B$, vol. 452, no. 3-4, pp. 347-354, 1999.

[36] B. Grzadkowski, Z. Hioki, K. Ohkuma, and J. Wudka, "Probing anomalous top-quark couplings induced by dim.6 operators at photon colliders," Nuclear Physics B, vol. 689, no. 1-2, pp. 108-126, 2004.

[37] S. M. Lietti and H. Murayama, " $C P$ violating $\mathrm{Zt} \bar{t}$ and $\gamma t \bar{t}$ couplings at a future $e^{+} e^{-}$collider," Physical Review D, vol. 62, no. 7, article 074003, 2000.

[38] Z. Hioki, T. Konishi, and K. Ohkuma, "Studying possible CPviolating Higgs couplings through top-quark pair productions at muon colliders," Journal of High Energy Physics, vol. 2007, no. 7, article 082, 2007.

[39] G. Valencia, "CP violation in top-quark pair production and decay," in Proceedings of The XIth International Conference on Heavy Quarks and Leptons - PoS(HQL 2012), p. 050, Prague, Czechia, December 2012.

[40] T. Han and Y. Li, "Genuine CP-odd observables at the LHC," Physics Letters B, vol. 683, no. 4-5, pp. 278-281, 2010.

[41] S. K. Gupta, A. S. Mete, and G. Valencia, " $C P$ violating anomalous top-quark couplings at the LHC," Physical Review D, vol. 80, no. 3, article 034013, 2009.

[42] T. Stelzer and W. F. Long, "Automatic generation of tree level helicity amplitudes," Computer Physics Communications, vol. 81, no. 3, pp. 357-371, 1994.

[43] J. Alwall, P. Demin, S. . Visscher et al., "MadGraph/MadEvent v4: the new web generation," Journal of High Energy Physics, vol. 2007, no. 9, article 028, 2007.

[44] J. Alwall, P. Artoisenet, S. de Visscher et al., "New developments in MadGraph/MadEvent," in AIP Conference Proceedings, vol. 1078, pp. 84-89, Seoul, Korea, 2009.

[45] T. Sjöstrand, S. Ask, J. R. Christiansen et al., "An introduction to PYTHIA 8.2," Computer Physics Communications, vol. 191, pp. 159-177, 2015.

[46] S. Ask, N. D. Christensen, C. Duhr et al., "From Lagrangians to events: computer tutorial at the MC4BSM-2012 workshop," https://arxiv.org/abs/1209.0297.

[47] R. D. Ball, V. Bertone, S. Carrazza et al., "Parton distributions with QED corrections," Nuclear Physics B, vol. 877, no. 2, pp. 290-320, 2013.

[48] The NNPDF collaboration, R. D. Ball, V. Bertone et al., "Parton distributions for the LHC run II," Journal of High Energy Physics, vol. 2015, no. 4, article 40, 2015.

[49] M. Tanabashi, K. Hagiwara, K. Hikasa et al., "Review of particle physics," Physical Review D, vol. 98, no. 3, article 030001, 2018.

[50] ATLAS collaboration, "ATLAS-CONF-2016-005”.

[51] CMS collaboration, CMS-PAS-TOP-16-005, CERN, Geneva, 2016.

[52] A. Mengarelli and ATLAS and CMS, "Top physics at LHC: from cross-section measurements to new physics searches," Nuovo Cimento C, vol. 40, no. 1, p. 5, 2017.

[53] M. Czakon, P. Fiedler, and A. Mitov, "Total top-quark pairproduction cross section at hadron colliders through $\mathcal{O}\left(\alpha_{S}^{4}\right)$," Physical Review Letters, vol. 110, no. 25, article 252004, 2013.

[54] H. Y. Zhou, " $C P$ violation in top quark pair production at hadron colliders," Physical Review D, vol. 58, no. 11, article 114002, 1998.

[55] S. Lee, "CP violating anomalous top-quark coupling in $\mathrm{p} \bar{p}$ collision at $\sqrt{ } s=1.96 \mathrm{TeV}$.
[56] S. M. Etesami and E. Darvish Roknabadi, "Probing the nonstandard top-gluon couplings through $t \bar{t} \gamma \gamma$ production at the LHC," Physical Review D, vol. 100, no. 1, article 015023, 2019.

[57] M. Jezabek, T. Nagano, and Y. Sumino, "Probe of $C P$ violation in $e^{+} e^{-} \longrightarrow t \bar{t}$ near threshold," Physical Review D, vol. 62, no. 1, article 014034, 2000.

[58] J. F. Kamenik, M. Papucci, and A. Weiler, "Constraining the dipole moments of the top quark," Physical Review D, vol. 85, no. 7, article 071501, 2012. 